\title{
How Much Should An Agent Know What Other Agents Are Doing in A Cooperative Team?
}

\author{
Yiming Ye \\ IBM T.J. Watson Research Center, Yorktown Heights, NY 10598, USA \\ yiming@watson.ibm.com
}

\begin{abstract}
Cooperation in a task oriented multi-agent team is important because a well designed cooperation strategy among a group of cooperating agents can lead to an increase of the group performance. A challenging research issue in cooperation is to assess how much and to what extent an agent should consider the actions and effects of other agents. In this paper, we address this issue in the context of an object search team. We first propose the concept of activity window to capture the view of an agent on the activities of the team. Then we pinpoint some criteria that can be used to determine whether it is beneficial for an agent to put an action of the team into its window. Finally, we present experimental results. ${ }^{1}$
\end{abstract}

\section{Introduction}

The coordination between an agent's actions and those of other cooperating agents is very important because an agent that considers the activities of other agents is usually better able to choose actions for itself that lead to favorable outcomes. It is obviously not a good strategy in a cooperative multi-agent team if the agents totally ignore one another, because the intended effects of one agent's action may already have been achieved by actions of other agents. On the other hand, it is also not a good strategy for each agent to keep track of all the activities of the other agents, since the effort required might detract from its own useful activities.

Thus, it is very important to study how and to what extent an agent should take into consideration the activities of other agents and what factors are important in deciding an agent's local coordination strategy. In this paper, we address this issue in the context of a multi-agent object search - a group of pan, tilt, and zoom cameras (or a group of robots) in the process of searching for a 3D object in

\footnotetext{
${ }^{1}$ Third International Conference on Multi-Agent Systems, 4 July - 7 July, 1998, La Villette, Paris, France
}

a $3 \mathrm{D}$ environment. The goal of the team is to maximize the probability of detecting the target within a given time constraint [1]. In [1], we study the complexity issue of the multiagent object search task.

\section{The Activity Window for a Given Agent}

In a cooperative object search team, when an agent executes an action, it will also broadcast the parameters of the action (i.e., the viewing direction and the viewing angle size) to all the other members of the team. (The communication time among agents for this purpose is small enough to be ignored). Thus, during the search process, an agent will continually receive information about the actions of other agents. The activity window $\mathbf{W}\left[a_{i}\right]$ for agent $a_{i}$ refers to the view of agent $a_{i}$ on the activities of the search team. The presence of an action $\mathbf{f}$ executed by agent $a_{j}$ in the activity window of agent $a_{i}$ (represented as $\mathbf{f} \in \mathbf{W}\left[a_{i}\right]$ ) indicates that agent $a_{i}$ updates its target distribution when $\mathbf{f}$ is executed by $a_{j}$. As we discuss in [1], an agent $a$ selects actions based on its knowledge $\mathbf{p}(a, c, \tau)(\forall c \in \Omega)$, where $\Omega$ is the search environment tessellated into small cells $c$, and $p(a, c, \tau)$ is the probability according to the belief of agent $a$ that the center of the target is within cell $c$ at time $\tau$. If the agent keeps track of every other agent's actions and updates its own knowledge accordingly, then its knowledge represents the true target distribution, and it can select high quality actions during the search process. Otherwise, its knowledge will be different from the true distribution, and it may not always be able to select good actions during the search process. The advantages of following the actions of the other agents during the search are clear, however the drawback is that it takes time to update the agent's knowledge. Thus, it is an important task for an agent in a multi-agent team to decide the extent to which it should pay attention to the activities of other agents, or in other words, to decide on the content of its activity window.

Good performance of the team depends on finding a balance between the benefits of shared knowledge and the price we have to pay. Here, we pinpoint some factors that may 
influence this balance. Let $n_{\text {update }}$ be the number of actions in the activity window of agent $a_{i}$, and assume that agent $a_{i}$ requires time $t_{\text {up date }}^{i}$ for each of these actions when updating its knowledge prior to choosing its own next action $\mathbf{f}$. Then there are three costs that need to be considered: (A) $t_{\text {select }}^{[i]}$, the time needed for agent $a_{i}$ to select an action; (B) $n_{\text {update }} \times t_{\text {update }}^{[i]}$, the time needed for agent $a_{i}$ to assess the activities of the team; $(C) t_{\text {execute }}^{[i]}$, the time needed for agent $a_{i}$ to execute an action. If the cost in executing an action $t_{\text {execute }}^{[i]}$ is relatively high compared to $t_{\text {update }}^{[i]}$, then it is worthwhile putting more actions in the activity window. In this way we can obtain more accurate knowledge about the target distribution and thus obtain high quality actions. However, if $t_{\text {ex ecute }}^{[i]}$ is relatively small compared to $t_{\text {update }}^{[i]}$, then it is not worth spending time following the activities of other agents, because the time can be devoted to executing more actions.

Let $T$ be the time used for search; $n_{i}$ be the total number of actions applied by agent $a_{i}$ within total time $T ; n_{i j}$ be the number of actions of agent $a_{j}$ which are considered by agent $a_{i}$. Then for a team in which every agent keeps track of all the actions of other agents, the following relations hold:

(A) $n_{i j} \leq n_{j}(j \neq i)$ and $n_{i i}=n_{i}-1$.

(B) $n_{i}\left(t_{\text {select }}^{[i]}+t_{\text {execute }}^{[i]}\right)+\left(n_{i 1}+\ldots+n_{\text {im }}\right) t_{\text {update }}^{[i]} \leq T$ (for $1 \leq i \leq m$ ).

Suppose $\Omega(\mathbf{f})$ and $\Omega[a]$ are the sets of cubes that can be checked by action $\mathbf{f}$ and agent $\boldsymbol{a}$ respectively. $\mathbf{R}_{a}$ and $\mathbf{R}_{a}\langle w, h\rangle$ are the distance ranges that can be checked by $\boldsymbol{a}$ and $\boldsymbol{a}$ with camera angle size $\langle w, h\rangle$ respectively. The following theorem and properties can be used by agent $a_{i}$ to select the contents of its activity window during the team search process.

Theorem: Suppose $\mathbf{f}$ is an action applied by agent $a_{j}$ during the search process. If $\Omega(\mathbf{f}) \bigcap \Omega\left[a_{i}\right]=\emptyset$, then there is no benefit from putting $\mathbf{f}$ into the activity window $\mathbf{W}\left[a_{i}\right]$ of agent $a_{i}$. In other words, the actions selected by agent $a_{i}$ will not be influenced whether $\mathbf{f}$ belongs to $\mathbf{W}\left[a_{i}\right]$ or not.

Property A Let $d_{i j}$ be the distance between agent $\boldsymbol{a}_{i}$ and agent $a_{j}$. If $d_{i j} \geq \mathbf{R}_{a_{i}}+\mathbf{R}_{a_{j}}$, then it is not necessary for agent $a_{i}$ to track actions executed by agent $a_{j}$.

Property $\mathbf{B}$ Let $d_{i j}$ be the distance between agent $a_{i}$ and agent $_{a_{j}}$. Let $\mathbf{f}$ be an action executed by agent $a_{j}$ with visual angle size $\langle w, h\rangle$. If $d_{i j} \geq \mathbf{R}_{a_{i}}+\mathbf{R}_{a_{j}}\langle w, h\rangle$, then it is not necessary for agent $\boldsymbol{a}_{i}$ to put $\mathbf{f}$ into its activity window.

\section{Experiments}

A $2 D$ simulation of a multi-agent object search system is implemented to test the influence of the activity window on the performance of the team and to examine various factors that should be considered in determining the content of the activity window. Figure 1(a)(b) shows the environment (the vertical rectangle is an obstacle) and the detection function used in the experiments. Figure 1(c)(d)(e)(f) show the environment (the shaded horizontal rectangle has a higher probability distribution) and the experimental results in testing the influence of the action execution time on the performance of the team when each agent keeps track of all the activities of the other agents and when each agent ignores any activities of other agents. The graphs show that when the action execution time is relatively small then the strategy of paying attention to the activities of the other agents does not perform as well as the strategy of simply ignoring the activities of the other agents. This situation changes gradually as the actions become more and more expensive. The second set of experiments (Figure $1(\mathrm{~g})(\mathrm{h})(\mathrm{i})$ ) test Property A. Figure 1(h) gives the delay in the execution starting time as a function of the action index of the team for the case where each agent attends to the activity broadcasts of fellow agents. Figure 1(i) compares the performance under the two strategies. The third set of experiments (Figure 1(j)(k)) test the benefits of selectively controlling the content of the activity window based on the criteria provided by Property A and Property B. Based on these criteria, it is beneficial for agents on the left to listen to each other and for agents on the right to listen to each other, but it is not useful for any agent on the left (right) to pay attention to any agent on the right (left). The experimental results shows this.

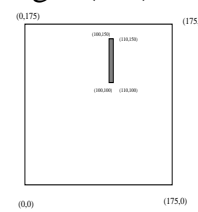

(a)

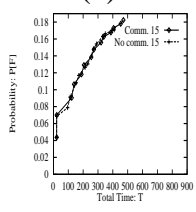

(e)

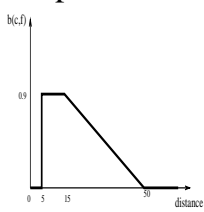

(b)

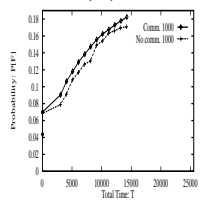

(f)

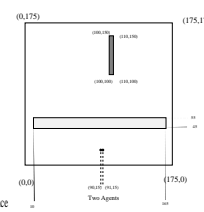

(c)

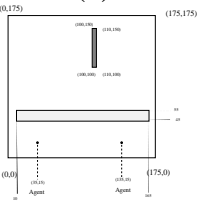

(g) (d)

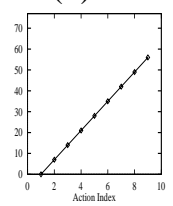

(h)

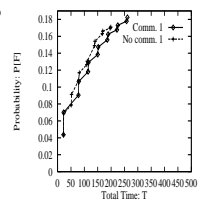

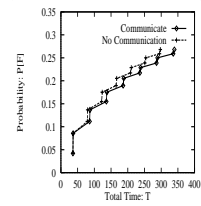

(i)

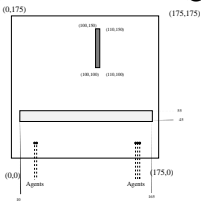

(j)

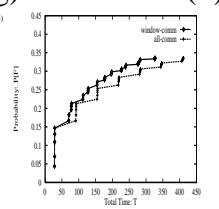

(k)
Figure 1. Experimental results.

\section{References}

[1] Y. Ye and J. K. Tsotsos. On the collaborative object search team: a formulation. In Distributed Artificial Intelligence Meets Machine Learning, Lecture Notes in Artificial Intelligence Vol. 1221, Gerhard WeiB Ed., pages 94-116, 1997. 